\title{
Lifetimes of Shockley electrons and holes at $\mathrm{Cu}(111)$
}

\author{
M. G. Vergniory, ${ }^{1}$ J. M. Pitarke, ${ }^{1,2}$ and S. Crampin ${ }^{3}$ \\ ${ }^{1}$ Materia Kondentsatuaren Fisika Saila, Zientzi Fakultatea, Euskal Herriko Unibertsitatea, 644 Posta kutxatila, E-48080 Bilbo, \\ Basque Country, Spain \\ ${ }^{2}$ Donostia International Physics Center (DIPC) and Unidad de Física Materiales CSIC-UPV/EHU, Manuel de Lardizabal Pasealekua, \\ E-20018 Donostia, Basque Country, Spain \\ ${ }^{3}$ Department of Physics, University of Bath, Bath BA2 7AY, United Kingdom \\ (Received 21 July 2005; revised manuscript received 15 September 2005; published 3 November 2005)
}

\begin{abstract}
A theoretical many-body analysis is presented of the electron-electron inelastic lifetimes of Shockley electrons and holes at the (111) surface of $\mathrm{Cu}$. For a description of the decay of Shockley states both below and above the Fermi level, single-particle wave functions have been obtained by solving the Schrödinger equation with the use of an approximate one-dimensional pseudopotential fitted to reproduce the correct bulk energy bands and surface-state dispersion. A comparison with previous calculations and experiment indicates that inelastic lifetimes are very sensitive to the actual shape of the surface-state single-particle orbitals beyond the $\bar{\Gamma}\left(\mathbf{k}_{\|}=0\right)$ point, which controls the coupling between the Shockley electrons and holes.
\end{abstract}

DOI: 10.1103/PhysRevB.72.193401

PACS number(s): 71.10.Ca, 72.15.Lh, 73.20.At, 68.37.Ef

A variety of metal surfaces, such as the (111) surfaces of the noble metals, are known to support a partially occupied band of Shockley surface states with energies near the Fermi level, ${ }^{1}$ whose dynamics have been the subject of longstanding interest. ${ }^{2-4}$ In particular, the lifetimes of excited holes at the band edge $\left(\mathbf{k}_{\|}=0\right)$ of these surface states have been investigated with high resolution angle-resolved photoemission $^{5-8}$ (ARP) and with the use of the scanning tunneling microscope (STM). ${ }^{9}$ STM techniques have also allowed the determination of the lifetimes of excited Shockley and image electrons over a range of energies above the Fermi level. ${ }^{10,11}$

Many-body calculations of the electron-electron $(e-e)$ inelastic lifetimes of excited holes at the surface-state band edge of the (111) surfaces of the noble metals $\mathrm{Cu}, \mathrm{Ag}$, and $\mathrm{Au}$, which were based upon the $G^{0} W$ one-dimensional scheme that had been introduced by Chulkov et al. ${ }^{12}$ to describe the lifetimes of image-potential states, ${ }^{13}$ showed considerable agreement with experiment. ${ }^{14,15}$ These calculations were then extended to treat the case of excited surface-state and surface-resonance electrons above the Fermi level. ${ }^{16,17}$ In order to account approximately for the potential variation in the plane of the surface, the original one-dimensional model potential, which had been introduced to describe surface states at the $\bar{\Gamma}$ point, was modified along with the introduction of a realistic effective mass for the dispersion curve of both bulk and surface states. Within this model, however, all Shockley states have the same effective mass, so the projected band structure is not correct, especially at energies above the Fermi level, as shown in Fig. 1.

In this paper, we present an approach that although at the $\bar{\Gamma}$ point is less sophisticated than the model used in Refs. 14-17 (i) basically reproduces the surface-state probability density of Refs. 14-17 at the band edge of the surface-state band in $\mathrm{Cu}(111)$ and (ii) has the merit that it reproduces, through the introduction of a $\mathbf{k}_{\|}$-dependent one-dimensional potential, the actual bulk energy bands and surface-state energy dispersion of Fig. 1, thereby allowing for a realistic description of the electronic orbitals beyond the $\bar{\Gamma}$ point. Adding the contribution from electron-phonon coupling, ${ }^{18}$ which is particularly important at the smallest excitation energies, our calculations of the lifetime broadening of excited Shockley electrons and holes in $\mathrm{Cu}(111)$ indicate that (i) there is good agreement with experiment at the surface-state band edge and (ii) at energies above the Fermi level the lifetime broadening is closer to experiment and very sensitive to the actual shape of the surface-state single-particle orbitals beyond the $\bar{\Gamma}$ point.

Let us consider a semi-infinite many-electron system that is translationally invariant in the plane of the surface (normal

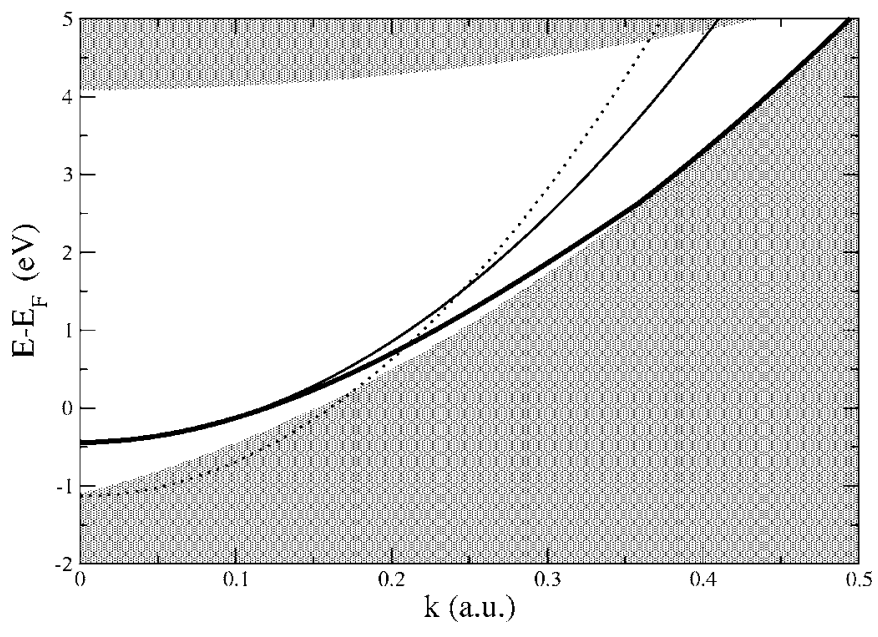

FIG. 1. Dispersion of the $\mathrm{Cu}(111)$ Shockley surface state (thick solid line), as obtained from three-dimensional $a b$ initio calculations. Shaded areas represent areas outside the band gap, where bulk states exist. The thin solid and dotted lines represent approximate energy dispersions of the Shockley surface state and the bottom of the projected band gap, respectively, as obtained from $\varepsilon$ $+k^{2} / 2 m$ with $\varepsilon=-0.44 \mathrm{eV}$ and $m=0.42$ in the case of the surface state (thin solid line) and $\varepsilon=-1.09 \mathrm{eV}$ and $m=0.22$ for the bottom of the projected band gap (thin dotted line). 
to the $z$ axis). The decay rate or inverse lifetime of a quasiparticle (electron or hole) that has been added to the system in the single-particle state $e^{i \mathbf{k}_{i} \mathbf{r}} \psi_{k_{i} E_{i}}(z)$ of energy $E_{i}$ is obtained as follows (we use atomic units, i.e., $e^{2}=\hbar=m_{e}=1$ ) ${ }^{19}$

$$
\Gamma_{k_{i}, E_{i}}=\mp 2 \int d z \int d z^{\prime} \psi_{k_{i} E_{i}}^{*}(z) \operatorname{Im} \Sigma\left(z, z^{\prime} ; k_{i}, E_{i}\right) \psi_{k_{i}, E_{i}}\left(z^{\prime}\right),
$$

where the $\mp$ sign in front of the integral should be taken to be minus or plus depending on whether the quasiparticle is an electron $\left(E_{i}>E_{F}\right)$ or a hole $\left(E_{i} \leqslant E_{F}\right)$, respectively, $E_{F}$ is the Fermi energy, $\mathbf{r}$ and $\mathbf{k}_{i}$ represent the position and wave vectors in the plane of the surface, and $\Sigma\left(z, z^{\prime} ; k_{i}, E_{i}\right)$ is the nonlocal self-energy operator.

To lowest order in a series-expansion of the self-energy $\Sigma\left(z, z^{\prime} ; k_{i}, E_{i}\right)$ in terms of the energy-dependent screened interaction $W\left(z, z^{\prime} ; k, E\right),{ }^{20,21}$ and replacing the interacting Green function $G\left(z, z^{\prime} ; k, E\right)$ by its noninteracting counterpart, one finds the following expression for the imaginary part of the so-called $G^{0} W$ self-energy:

$$
\begin{aligned}
\operatorname{Im} \Sigma\left(z, z^{\prime} ; k_{i}, E_{i}\right)= & \frac{1}{\pi} \int_{0}^{\left|E_{i}-E_{F}\right|} d E \int \frac{d \mathbf{q}}{(2 \pi)^{3}} \\
& \times \operatorname{Im} G^{0}\left(z, z^{\prime} ; k_{f}, E_{f}\right) \operatorname{Im} W\left(z, z^{\prime} ; q, E\right) .
\end{aligned}
$$

Here, $\mathbf{k}_{f}=\mathbf{k}_{i}-\mathbf{q}, E_{f}=E_{i}-E$, and $G^{0}\left(z, z^{\prime} ; k, E\right)$ is the noninteracting Green function

$$
G^{0}\left(z, z^{\prime} ; k, E\right)=2 \frac{\psi_{k, E}^{+}\left(z^{>}\right) \psi_{k, E}^{-}\left(z^{<}\right)}{\left[\psi_{k, E}^{+}, \psi_{k, E}^{-}\right](z)},
$$

where $z^{<}\left(z^{>}\right)$is the lesser (greater) of $z$ and $z^{\prime}$, and $[f, g](z)$ is the Wronskian

$$
[f, g](z)=f(z) g^{\prime}(z)-f^{\prime}(z) g(z) .
$$

The functions $\psi_{k, E}^{ \pm}(z)$ are solutions of the single-particle Schrödinger equation

$$
-(1 / 2) \psi_{k, E}^{\prime \prime}+V_{k}(z) \psi_{k, E}(z)=\left(E-k^{2} / 2\right) \psi_{k, E}(z)
$$

regular at $\pm \infty$, with $V_{k}(z)$ being a momentum-dependent onedimensional effective potential that we fit to the projected surface band structure. We use

$$
V_{k}(z)= \begin{cases}U_{k}+2 V_{k} \cos \left(2 \pi z / a_{s}\right), & z<z_{k} \\ \Phi, & z>z_{k},\end{cases}
$$

where $U_{k}$ and $V_{k}$ are fitted to the bulk energy bands (which we have obtained from three-dimensional $a b$ initio calculations), $a_{s}=2.08 \AA$ represents the interlayer spacing, $\Phi$ $=4.94 \mathrm{eV}$ is the experimentally determined work function, and the matching plane $z_{k}$ is chosen to give the correct surface-state dispersion represented in Fig. 1 by a thick solid line. ${ }^{22}$

In the random-phase approximation (RPA), ${ }^{23}$ the screened interaction $W\left(z, z^{\prime} ; q, E\right)$ is obtained from the knowledge of the noninteracting density-response function $\chi^{0}\left(z, z^{\prime} ; q, E\right)$ by solving the following integral equation:

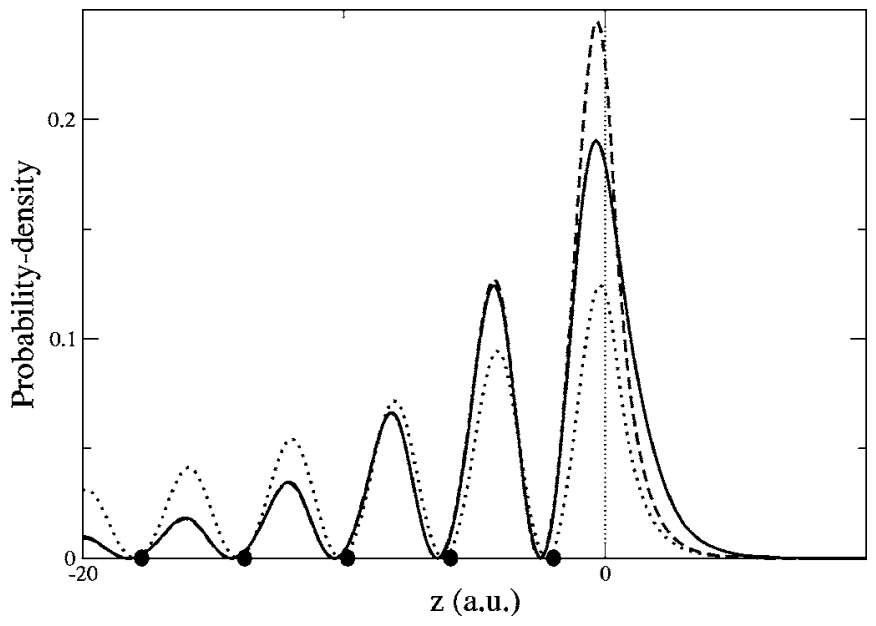

FIG. 2. Probability-density $\left|\psi_{k, E}\right|^{2}$ of the Shockley surface state at the center of the surface Brillouin zone of $\mathrm{Cu}(111)$, as obtained within two different models: the model presented here (dashed line) and the model reported in Ref. 12 (solid line). The dotted line represents the probability-density that we obtain at $k=0.2$ a.u.. Full circles represent the atomic positions of $\mathrm{Cu}$ in the (111) direction. The geometrical (jellium) electronic edge $(z=0)$ has been chosen to be located half an interlayer spacing beyond the last atomic layer.

$$
\begin{aligned}
W\left(z, z^{\prime} ; q, E\right)= & v\left(z, z^{\prime} ; q\right)+\int d z_{1} \int d z_{2} \\
& \times v\left(z, z_{1} ; q\right) \chi^{0}\left(z_{1}, z_{2} ; q, E\right) W\left(z_{2}, z^{\prime} ; q, E\right),
\end{aligned}
$$

where $v\left(z, z^{\prime} ; q\right)$ represents the two-dimensional Fourier transform of the bare Coulomb interaction. The results presented below have been obtained by using in $\chi^{0}\left(z, z^{\prime} ; q, E\right)$ the eigenfunctions and eigenvalues of the one-dimensional Hamiltonian of Ref. 12 with all effective masses set equal to the free-electron mass. We have also used the eigenfunctions and eigenvalues of a single-particle jellium-surface KohnSham Hamiltonian (in the local-density approximation) with $r_{s}=2.67,{ }^{24}$ and we have found that the surface-state lifetimes are rather insensitive to whether one or the other choice is employed.

The abrupt step model potential of Eq. (6), which does not account for the image tail outside the surface, could not possibly be used to describe image states. However, Shockley surface states are known to be rather insensitive to the actual shape of the potential outside the surface; indeed, the model potential of Eq. (6) is found to yield a surface-state probability-density $\left|\psi_{k_{i}, E_{i}}\right|^{2}$ at the band edge of the Shockley surface-state band of $\mathrm{Cu}(111)\left(k_{i}=0\right)$ that is in reasonably good agreement with the more realistic surface-state probability density used in Refs. 14-17, as shown in Fig. 2. Both probability-densities coincide within the bulk, although our approximate probability-density appears to be slightly more localized near the surface, as expected. ${ }^{25}$ Nevertheless, we find that decay rates of an excited hole at $\bar{\Gamma}$ based on the use of these two models to describe the wave function $\psi_{k_{i}, E_{i}}$ entering Eq. (1) agree within less than $1 \mathrm{meV}$. Differences be- 
TABLE I. $G^{0} W$ decay rates, in linewidth units $(\mathrm{meV})$, of an excited hole at the band edge of the Shockley surface-state band of $\mathrm{Cu}(111)\left(E_{i}=-0.44 \mathrm{eV}\right.$ and $\left.k_{i}=0\right) . \Gamma_{\text {inter }}$ and $\Gamma_{\text {intra }}$ represent interband and intraband contributions to the $e$-e decay rate $\Gamma_{e-e}$. The total decay rate $\Gamma_{\text {total }}$ includes the $e$-ph decay rate of $7 \mathrm{meV}$ reported in Ref. 18. The full calculation represents the result we have obtained by using in Eq. (3) the actual $k$-dependent model single particle wave function $\psi_{k, E}$. The approximate calculation represents the result we have obtained by replacing all surface-state wave functions with $\mathbf{k} \neq 0$ by that at the $\bar{\Gamma}$ point. The third row represents the calculations reported in Refs. 14 and 15. The experimental linewidth has been taken from the STM measurements reported in Ref. 14.

\begin{tabular}{lcccc}
\hline \hline Calculation & $\Gamma_{\text {inter }}$ & $\Gamma_{\text {intra }}$ & $\Gamma_{e-e}$ & $\Gamma_{\text {total }}$ \\
\hline Full & 10 & 9 & 19 & 26 \\
Approximate & 10 & 23 & 33 & 40 \\
Refs. 14 and 15 & 6 & 19 & 25 & 32 \\
Experiment & & & & 24 \\
\hline \hline
\end{tabular}

tween our new calculations, which are based on the use of the $k$-dependent model potential of Eq. (6), and those reported previously, ${ }^{14-17}$ are due to our more realistic description of the band structure and surface-state wave functions beyond the $\bar{\Gamma}$ point.

First of all, we consider an excited hole at the band edge of the Shockley surface-state band of $\mathrm{Cu}(111)$, i.e., with $E_{i}$ $=-0.44 \mathrm{eV}$ and $k_{i}=0$ (see Fig. 1). The decay of this excited quasiparticle may proceed either through the coupling with bulk states (interband contribution) or through the coupling, within the surface-state band itself, with surface states of different wave vector $\mathbf{k}$ parallel to the surface (intraband contribution). In order to investigate the impact of the actual shape of the surface-state wave functions with $\mathbf{k} \neq 0$ on the decay of the surface-state hole at $\bar{\Gamma}$, we have compared in Table I the decay rates that we have calculated either by using in Eq. (3) the actual $k$-dependent model wave function $\psi_{k, E}$ (full calculation) or by replacing all surface-state wave functions $\psi_{k, E}$ with $\mathbf{k} \neq 0$ by that at the $\bar{\Gamma}$ point (approximate calculation). This comparison shows that the penetration of the actual $k$-dependent surface-state wave functions $\psi_{k, E}$ being larger than at $\bar{\Gamma}$ (compare the dashed and dotted lines of Fig. 1) yields a reduction in the decay rate from $33 \mathrm{meV}$ to $19 \mathrm{meV}$, which is due to the fact that the coupling of the surface-state hole at $\bar{\Gamma}$ with actual surface states of different wave vector $\mathbf{k}$ (intraband contribution) is smaller than the coupling that would take place with surface-state orbitals that do not change with $\mathbf{k}$. The difference between our predicted surface-state lifetime broadening of $19 \mathrm{meV}$ and that reported before $\left(\tau^{-1}=25 \mathrm{meV}\right)$ (Refs. 14 and 15) is entirely due to our more accurate description of (i) the projected band structure and (ii) the wave-vector dependence of the surface-state wave functions $\psi_{k, E}(z)$ entering the evaluation of the Green function of Eq. (3).

We have also carried out a full calculation of the decay of an excited hole at $\bar{\Gamma}$ but replacing the actual surface-state wave vector $\mathbf{k}_{f}$ entering Eq. (2) by the wave vector that

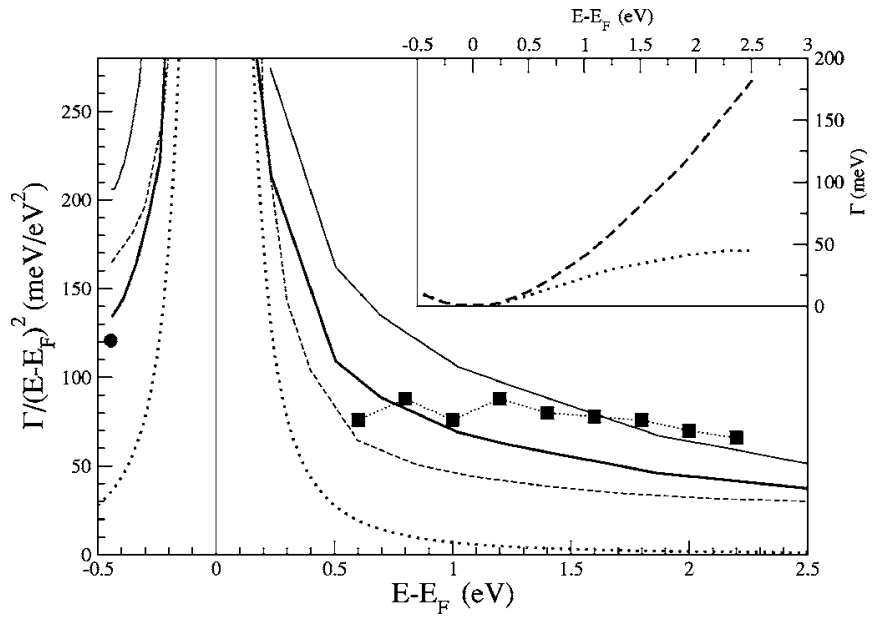

FIG. 3. Scaled total linewidth $\Gamma_{\text {total }} /\left(E_{i}-E_{F}\right)^{2}$ of Shockley surface-state electrons and holes in $\mathrm{Cu}(111)$ (including the $e$-ph contribution $\Gamma_{e \text {-ph }}=7 \mathrm{meV}$ ) versus the surface-state energy $E_{i}$, as obtained from Eqs. (1)-(7) (thick solid line), from Eqs. (1)-(7) but replacing all surface-state wave functions with $\mathbf{k} \neq 0$ by those at the $\bar{\Gamma}$ point (thin solid line), and from Ref. 16 (thin dashed line). The dotted line represents the energy-independent $e$-ph contribution $\Gamma_{e-\mathrm{ph}}=7 \mathrm{meV}$. The dotted line with squares represents the STM measurements reported in Ref. 10, multiplied by a factor of 2 to correct for an error in the phase coherence length used in that work. The solid circle represents the experimentally determined linewidth of an excited hole at the $\bar{\Gamma}$ point, as reported in Ref. 14. Separate unscaled interband $\left(\Gamma_{\text {inter }}\right)$ and intraband $\left(\Gamma_{\text {intra }}\right)$ contributions to the total linewidth $\left(\Gamma_{\text {total }}=\Gamma_{\text {intra }}+\Gamma_{\text {inter }}+\Gamma_{e \text {-ph }}\right)$ are represented in the inset by dashed and dotted lines, respectively.

would correspond to a parabolic surface-state dispersion of the form dictated by the thin solid line of Fig. 1, and we have found that the linewidth is reduced (as expected, since the parabolic dispersion results in fewer final states) by less than $\sim 1 \mathrm{meV}$. However, if one further replaces our wave-vector dependent surface-state orbitals entering Eq. (2) by their less accurate counterparts used previously, ${ }^{14,15}$ the lifetime broadening is increased considerably (from 19 to $25 \mathrm{meV}$ ), showing the important role that the actual coupling between initial and final states plays in the surface-state decay mechanism.

Our model, which correctly reproduces the behavior of $s-p$ valence states, does not account for the presence of $d$ electrons with energies a few electronvolts below the Fermi level. The screening of $d$ electrons is known to play a crucial role in the decay mechanism of bulk states. ${ }^{26}$ However, in the case of Shockley holes, whose decay is dominated by intraband transitions that are associated with very small values of the momentum transfer, the screening of $d$ electrons is expected to reduce the lifetime broadening only very slightly, ${ }^{27}$ and will not be included in the present work. Adding to our estimated $e-e$ linewidth of $19 \mathrm{meV}$ the electron-phonon (e-ph) linewidth of $7 \mathrm{eV}$ reported in Ref. 18, we find $\Gamma_{\text {total }}$ $=26 \mathrm{meV}$ in close agreement with the experimentally measured linewidth of $24 \mathrm{meV}$, as shown in Table I.

In Fig. 3, we show our full calculation (thick solid line) of the inelastic linewidth $\left(\Gamma_{\text {total }}=\Gamma_{e-e}+\Gamma_{e-\mathrm{ph}}\right)$ of excited Shockley holes and electrons in $\mathrm{Cu}(111)$ with energies $E_{i}$ below 
and above the Fermi level. Also shown are separate interband and intraband contributions to the linewidth (inset), the approximate calculation that we have carried out by replacing all surface-state wave functions with $\mathbf{k} \neq 0$ by the surface-state wave function at $\bar{\Gamma}$ (thin solid line), and the calculations reported in Ref. 16 (thin dashed line). The lifetime broadening of excited Shockley electrons in $\mathrm{Cu}(111)$ was studied with the STM by Bürgi et al. ${ }^{10}$ However, it has been argued recently ${ }^{28}$ that due to an error in the identification of the phase coherence length in the measured quantum interference patterns the linewidths reported in Ref. 10 should be doubled. These doubled values have been represented in Fig. 3 by solid squares. The experimentally determined inelastic linewidth of an excited Shockley hole at the $\bar{\Gamma}$ point $^{14}$ is represented by a solid circle.

Figure 3 shows that a correct description of the wavevector dependent surface-state wave functions reduces the coupling of holes and electrons within the Shockley band, thereby bringing the lifetime broadening into closer agreement with experiment. A comparison between our full calculations and experiment shows that there is close agreement at the surface-state band edge (at $E-E_{F}=-0.44 \mathrm{eV}$ ) and there is also reasonable agreement at energies above the Fermi level. At energies where the surface-state band merges into the continuum of bulk states, however, our calculated linewidths are still too low, which might be a signature of the need of a fully three-dimensional description of the surface band structure. We also note that differences between the calculations reported here and those reported previously ${ }^{16}$ indicate that inelastic lifetimes are very sensitive to the actual shape of the surface-state single-particle orbitals beyond the $\bar{\Gamma}$ point. The linewidths reported here are smaller (larger) for excited holes (electrons) below (above) the Fermi level, thus bringing the theretical predictions closer to experiment.

In summary, we have presented a $G^{0} W$ one-dimensional scheme to calculate the inelastic lifetime broadening of excited Shockley electrons and holes in $\mathrm{Cu}(111)$, which is based on a realistic description of the projected bulk energy bands and the surface-state orbitals beyond the $\bar{\Gamma}$ point. Adding the contribution from electron-phonon coupling, ${ }^{18}$ which is particularly important at the smallest excitation energies, our calculations indicate that there is reasonable agreement with experiment, especially at low excitation energies. The screening of $d$ electrons, not included in this work, is expected to reduce the lifetime broadening only very slightly, at least at the hot-electron energies nearest to the Fermi level.

The authors thank E. V. Chulkov, P. M. Echenique, and J. E. Inglesfield for enjoyable discussions and a careful reading of the manuscript. M.G.V. and J.M.P. acknowledge partial support by the University of the Basque Country, the Basque Unibertsitate eta Ikerketa Saila, the MCyT, and the EC 6th framework Network of Excellence NANOQUANTA (Grant No. NMP4-CT-2004-500198).
${ }^{1}$ J. E. Inglesfield, Rep. Prog. Phys. 45, 223 (1982).

${ }^{2}$ R. Matzdorf, Surf. Sci. Rep. 30, 153 (1998).

${ }^{3}$ P. M. Echenique et al., Chem. Phys. 251, 1 (2000).

${ }^{4}$ P. M. Echenique et al., Surf. Sci. Rep. 52, 219 (2004).

${ }^{5}$ S. D. Kevan, Phys. Rev. Lett. 50, 526 (1983).

${ }^{6}$ S. D. Kevan and R. H. Gaylord, Phys. Rev. B 36, 5809 (1987).

${ }^{7}$ B. A. McDougall et al., Phys. Rev. B 51, R13891 (1995).

${ }^{8}$ W. Schattke and M. A. Van Hove, Solid-State Photoemission and Related Methods (Wiley/VCH, Weinheim, 2003).

${ }^{9}$ J. Li et al., Phys. Rev. Lett. 81, 4464 (1998).

${ }^{10}$ L. Bürgi et al., Phys. Rev. Lett. 82, 4516 (1999).

${ }^{11}$ P. Wahl et al., Phys. Rev. Lett. 91, 106802 (2003).

${ }^{12}$ E. V. Chulkov et al., Surf. Sci. 391, L1217 (1997).

${ }^{13}$ E. V. Chulkov et al., Phys. Rev. Lett. 80, 4947 (1998).

${ }^{14}$ J. Kliewer et al., Science 288, 1399 (2000).

${ }^{15}$ P. M. Echenique et al., Appl. Phys. A: Mater. Sci. Process. 71, 503 (2000).

${ }^{16}$ P. M. Echenique et al., Prog. Surf. Sci. 67, 271 (2001).

${ }^{17}$ L. Vitali et al., Surf. Sci. 523, L47 (2003).

${ }^{18}$ A. Eiguren et al., Phys. Rev. Lett. 88, 066805 (2002).

${ }^{19}$ M. Nekovee and J. M. Pitarke, Comput. Phys. Commun. 137,
$123(2001)$

${ }^{20}$ L. Hedin and S. Lundqvist, Solid State Phys. 23, 1 (1969).

${ }^{21}$ F. Aryasetiawan and O. Gunnarsson, Rep. Prog. Phys. 61, 237 (1998).

${ }^{22}$ At the $\bar{\Gamma}$ point $(k=0)$, one finds $U_{k}=-7.00 \mathrm{eV}$ (with respect to the Fermi level), $2 V_{k}=5.17 \mathrm{eV}$, and $z_{k}=0.18 \AA$ (with respect to the jellium edge). At $k=0.5$ a.u., we find $U_{k}=-4.24 \mathrm{eV}, 2 V_{k}$ $=0.19 \mathrm{eV}$, and $z_{k}=0.37 \AA$.

${ }^{23}$ A. L. Fetter and J. D. Wallecka, Quantum Theory of ManyParticle Systems (McGraw-Hill, New York, 1964).

${ }^{24}$ The valence electron density $n$ of the solid is usually characterized by the density parameter $r_{s}=(3 / 4 \pi n)^{1 / 3} / a_{0}, a_{0}$ being the Bohr radius $a_{0}=0.529 \AA$.

${ }^{25}$ For the overlap integral we have found $\left\langle\psi_{1} \mid \psi_{2}\right\rangle=0.99, \psi_{1}$ and $\psi_{2}$ being the Shockley probability amplitudes leading to the probability-densities represented in Fig. 2 by solid and dashed lines.

${ }^{26}$ I. Campillo et al., Phys. Rev. Lett. 83, 2230 (1999).

${ }^{27}$ A. García-Lekue et al., Phys. Rev. B 68, 045103 (2003).

${ }^{28}$ S. Crampin et al., Phys. Rev. Lett. 95, 029701 (2005). 